\title{
Avaliação de Trichogramma spp. para o controle de Trichoplusia ni
}

\author{
André Malacarne Milanez ${ }^{(1)}$, Dirceu Pratissoli ${ }^{(2)}$, Ricardo Antonio Polanczyk ${ }^{(2)}$, Adeney de Freitas Bueno(3) \\ e Camila Borges Antonio Tufik ${ }^{(2)}$
}

\begin{abstract}
(1)Universidade Federal Rural de Pernambuco, Departamento de Agronomia e Entomologia, Avenida Dom Manoel de Medeiros, s/no, Dois Irmãos, CEP 52171-900 Recife, PE. E-mail: andremalak@hotmail.com (2)Universidade Federal do Espírito Santo, Centro de Ciências Agrárias, Departamento de Produção Vegetal, Alto Universitário, s/no, CEP 29500-000 Alegre, ES. E-mail: pratissoli@cca.ufes.br, ricardo@cca.ufes.br, camila_btufik@hotmail.com ${ }^{(3)}$ Embrapa Soja, Caixa postal 231, CEP 86001-970 Londrina, PR. E-mail: adeney@pq.cnpq.br
\end{abstract}

Resumo - O objetivo deste trabalho foi avaliar as características biológicas dos parasitoides Trichogramma acacioi, T. atopovirilia, T. marandobai, T. demoraesi, T. exiguum (duas linhagens) e T. pretiosum (seis linhagens), criados em ovos de Trichoplusia ni. O experimento foi conduzido em delineamento inteiramente casualizado, com 15 repetições. As características biológicas avaliadas foram: parasitismo, viabilidade, razão sexual e número de indivíduos por ovo. A percentagem de parasitismo variou entre 7,66 e 53\%, com maior valor observado para a linhagem Tspd de Trichogramma pretiosum, e o menor para a linhagem Trecife de T. pretiosum. Não houve diferença significativa dessa espécie quanto à viabilidade, que ficou acima de $85 \%$. A razão sexual variou de 0,75 a 1, e a linhagem Tspd apresentou o menor valor. O número de parasitoides por ovo variou entre 2,39 para T. marandobai (linhagem Tm1) e 1,34 para T. exiguum (linhagem Te1). A espécie que apresentou o melhor desempenho em laboratório foi T. pretiosum (Tspd), com os maiores valores observados na percentagem de parasitismo e na emergência.

Termos para indexação: Brassicaceae, controle biológico, lagarta mede-palmo, parasitismo, parasitoide de ovos.

\section{Evaluation of Trichogramma spp. for the control of Trichoplusia ni}

\begin{abstract}
The objective of this study was to evaluate the biological characteristics of the parasitoids Trichogramma acacioi, T. atopovirilia, T. marandobai, T. demoraesi, T. exiguum (two lines), and T. pretiosum (six lines), reared on eggs of Trichoplusia ni. The experiment was carried out in a completely randomized design, with 15 replicates. The biological characteristics evaluated were: parasitism, viability, sexual ratio and number of individuals per egg. The parasitism percentage varied between 7.66 and 53\%, whith highest value observed for Trichogramma pretiosum Tspd line, and the lowest for the T. pretiosum Trecife line. There was no significant difference for the viability, which remained higher than $85 \%$. The sex ratio varied from 0.75 to 1 , and Tspd line showed the lowest value. The number of individuals per egg varied between 2.39 for T. marandobai (Tm1 line) and 1.34 for T. exiguum (Te1 line). T. pretiosum (Tspd line) was the species with the best performance in laboratory with the highest values observed in parasitism and in viability.
\end{abstract}

Index terms: Brassicaceae, biological control, Cabbage looper, parasitism, egg parasitoids.

\section{Introdução}

A lagarta mede-palmo Trichoplusia ni (Hübner) (Lepdoptera: Noctuidae) é uma praga polífaga, que tem como hospedeiros: brássicas, tomate, pimentão, pepino, melancia, beterraba, alface, algodão, soja; e plantas daninhas como alface selvagem, dente-de-leão e olerícolas em cultivo protegido (Jost \& Pitre, 2002; Janmaat \& Myers, 2003). A presença de T. ni em campo, durante o ano todo, se deve ao fato de essa espécie alimentar-se de uma grande variedade de hospedeiros, bem como à ocorrência de populações da espécie que se tornaram resistentes ao uso de inseticidas (Liu et al., 2002; Castells \& Berenbaum, 2008).

Para o manejo fitossanitário dessa praga, deve-se priorizar a associação de diversos métodos de controle, para obtenção de resultados satisfatórios e mais duradouros, pois o impacto ambiental do uso de agrotóxicos pode levar à ressurgência e à resistência da praga, à destruição dos inimigos naturais

Pesq. agropec. bras., Brasília, v.44, n.10, p.1219-1224, out. 2009 
e polinizadores, além de causar contaminação do ambiente (Hart \& Pimentel, 2002). Assim, os inimigos naturais são importantes agentes de controle biológico, pois mantêm as populações de insetos-praga abaixo do nível de dano econômico, como evidenciado no agroecossistema das brássicas (Godin \& Boivin, 1998). A utilização do controle biológico com parasitoides do gênero Trichogramma, quando bem implantado, pode ser uma alternativa viável ao controle químico.

Parasitoides de ovos do gênero Trichogramma têm sido utilizados no controle de pragas, pois são de fácil multiplicação massal em laboratório, com custo viável (Parra, 2002), além de promover o controle da praga antes que ocorra dano à cultura (Pratissoli et al., 2007). Este fato é desejável na cultura das brássicas, pois elas perdem valor ou, muitas vezes, são impróprias para comercialização quando apresentam danos causados pelo ataque da praga.

O sucesso no controle de pragas, com o uso do parasitoide Trichogramma, está relacionado à escolha correta da espécie e linhagem a serem utilizadas, pois embora seja classificado como parasitoide generalista, resultados de pesquisa demonstram que as espécies e linhagens podem apresentar afinidade por determinados hospedeiros, em razão do comportamento de busca orientado por estímulos, das características nutricionais e morfológicas do ovo, além de fatores abióticos como condições climáticas (Vinson, 1997; Pratissoli \& Parra, 2001; Mansfield \& Mills, 2004).

O objetivo deste trabalho foi avaliar as características biológicas de espécies e linhagens de Trichogramma para selecionar a de maior potencial para o manejo fitossanitário de Trichoplusia ni.

\section{Material e Métodos}

O experimento foi conduzido no Núcleo de Desenvolvimento Científico e Tecnológico em Manejo Fitossanitário (Nudemafi), do Centro de Ciências Agrárias, da Universidade Federal do Espírito Santo, Alegre, ES, em câmaras climatizadas ajustadas a $25 \pm 1{ }^{\circ} \mathrm{C}, 70 \pm 10 \%$ de temperatura e umidade relativa, respectivamente, e fotófase de 12 horas.

As lagartas de Trichoplusia ni foram coletadas em culturas de repolho e couve-flor, no Município de Domingos Martins, ES, e criadas até a fase de pupa, em couve. Os adultos foram mantidos em gaiolas (60x50x50 cm) e, como local de oviposição das fêmeas, foram utilizadas folhas de couve, acondicionadas com o pecíolo em um frasco de vidro de $300 \mathrm{~mL}$ com água. Aos adultos, foi oferecida, também, solução de mel a $10 \%$, em frascos de $20 \mathrm{~mL}$, com chumaço de algodão em contato com a solução. $\mathrm{O}$ alimento foi renovado a cada 48 horas. Diariamente, pela manhã, a folha de couve era substituída por uma nova, e as folhas com os ovos eram acondicionadas em recipiente de plástico. Lagartas recém-eclodidas foram transferidas da folha de couve para uma dieta artificial à base de feijão, levedura de cerveja e germe de trigo (Greene et al., 1976). A dieta foi acondicionada em tubos de vidro $(8 \times 2,5 \mathrm{~cm})$ com três lagartas cada, que se alimentaram nesse meio até a fase de pupa.

O hospedeiro alternativo, Anagasta kuehniella (Zeller) (Lepidoptera: Pyralidae), foi criado de acordo com a metodologia desenvolvida pelo laboratório do Nudemafi, em dieta à base de farinha de trigo integral (60\%), farinha de milho (37\%) e levedura de cerveja (3\%). A criação e manutenção de $A$. kuehniella foram feitas em caixas de plástico $(30 \times 25 \times 10 \mathrm{~cm})$, onde foi depositada a dieta previamente homogeneizada. Cerca de $0,3 \mathrm{~g}$ de ovos foram distribuídos sobre a dieta, que serviu de substrato alimentar para as traças. Com a emergência dos adultos, iniciou-se a coleta diária, com um aspirador de pó adaptado. As mariposas coletadas foram transferidas para vasilhas de plástico com telas de náilon no interior, dobradas em ziguezague, que serviram de substrato para a oviposição. A extremidade superior da gaiola foi fechada com tecido tipo filó. Os ovos foram coletados diariamente, por cinco dias, e posteriormente armazenados e conservados em geladeira a $4 \pm 1^{\circ} \mathrm{C}$, pelo período máximo de 20 dias.

As espécies de Trichogramma foram criadas e multiplicadas em ovos do hospedeiro alternativo A. kuehniella. Os ovos foram colados em retângulos de cartolina azul celeste $(8 \times 2 \mathrm{~cm})$, com goma arábica diluída a $30 \%$, e foram posteriormente inviabilizados, pela exposição à lâmpada germicida por 45 min (Stein \& Parra, 1987). Em uma das extremidades das cartelas, foram anotados a data de parasitismo e o código de identificação da espécie. Após serem inviabilizados, os ovos foram oferecidos às fêmeas dos parasitoides, em tubos de vidro $(8,5 \times 2,5 \mathrm{~cm})$, mantidos em câmaras climatizadas.

As espécies e linhagens utilizadas no experimento foram: Trichogramma acacioi Brun Moraes \& Soares; T. atopovirilia Oatman \& Platner; T. marandobai 
Brun Moraes \& Soares; T. demoraesi Nagaraja; duas linhagens de T. exiguum Pinto \& Platner e seis linhagens de $T$. pretiosum Riley (Hymenoptera: Trichogrammatidae) (Tabela 1).

Fêmeas recém-emergidas dos parasitoides foram individualizadas em tubos de vidro $(8,5 \times 2,5 \mathrm{~cm})$, vedados com filme de PVC, e alimentadas com gotículas de mel puro. Foram oferecidos 20 ovos de Trichoplusia ni com até 24 horas de desenvolvimento embrionário, que foram retirados individualmente da postura, com o auxílio de um microscópio estereoscópio e um pincel umedecido, e fixados à cartolina azul celeste de $3 \times 2 \mathrm{~cm}$ com goma arábica a 10\%. Após 24 horas de exposição aos ovos, os parasitoides foram removidos. As cartelas com os ovos foram mantidas em tubos de vidro $(8,5 \times 2,5 \mathrm{~cm})$ vedados com filme de PVC, nas condições de temperatura, umidade e fotófase previamente mencionados. Diariamente, foram removidas as lagartas recém-eclodidas dos ovos não parasitados.

Após a morte dos descendentes, foram avaliados o número de ovos parasitados, os ovos com orifício e o número de machos e fêmeas. Posteriormente, o número de ovos parasitados e a viabilidade foram expressos em percentagem; o número total de parasitoides foi dividido pelo número de ovos com orifício, para se determinar o número de parasitoides por ovo; e a razão sexual foi determinada pelo número de fêmeas, em relação ao total de indivíduos na população.

$\mathrm{O}$ delineamento experimental foi inteiramente casualizado, com 15 repetições. Os dados de percentagem de parasitismo e percentagem de emergência foram transformados para $\operatorname{arcsen}(\mathrm{x} / 100)^{0,5}$; os dados de razão sexual e número de indivíduos por ovo foram transformados para $(\mathrm{x}+0,5)^{0,5}$ submetidos à análise de variância; as médias foram comparadas pelo teste de Scott-Knott, a 5\% de probabilidade, com o uso do programa computacional SAEG.

\section{Resultados e Discussão}

Houve diferença significativa entre as médias de percentagem de parasitismo, das espécies e linhagens de Trichogramma avaliadas (Tabela 2). A linhagem Tspd de T. pretiosum obteve a maior percentagem de parasitismo (53\%) de ovos de Trichoplusia ni. A linhagem Tac2 de Trichogramma acacioi, a Tat1 de $T$. atopovirilia e a Tp8 de $T$. pretiosum foram estatisticamente semelhantes, com 36,31 e $26,6 \%$ de ovos parasitados, respectivamente, e foram superiores às demais espécies e linhagens que apresentaram taxas inferiores a $21 \%$ de parasitismo.

O gênero Trichogramma é classificado como generalista; entretanto, os resultados obtidos no presente trabalho mostram a importância da seleção de espécies e linhagens desse parasitoide no controle de pragas, uma vez que há diferenças no parasitismo entre espécies distintas, e entre linhagens da mesma espécie. Essas variações ocorrem em razão do comportamento de busca e aceitação do hospedeiro, que é orientado pelas características nutricionais e morfológicas do ovo como tamanho e forma, e espessura e rigidez do córion (Vinson, 1997; Pratissoli \& Parra, 2001; Roriz et al., 2006). Diferenças no potencial de parasitismo de espécies de Trichogramma já foram relatadas por Cônsoli \& Parra (1999), que observaram diferenças na capacidade de postura de linhagens de T. pretiosum em ovos artificiais; e por Pratissoli \& Parra (2001) que, ao avaliar seis linhagens de $T$. pretiosum provenientes dos estados do Espírito Santo, Pernambuco, Minas

Tabela 1. Espécies e linhagens de Trichogramma, com os respectivos hospedeiros naturais, culturas e locais de coleta.

\begin{tabular}{lcccc}
\hline Espécie & Linhagem & Hospedeiro & Cultura & Localidade \\
\hline T. acacioi & Tac2 & Nipteria panacea & Abacate & Venda Nova do Imigrante, ES \\
T. atopovirilia & Tat1 & Helicoverpa zea & Milho & Sete Lagoas, MG \\
T. demoraesi & $\mathrm{Td} 1$ & - & - & Montes Claros, MG \\
T. exiguum & $\mathrm{Te} 1$ & - & Tomate & Muniz Freire, ES \\
T. exiguum & $\mathrm{Te} 3$ & - & Tomate & Alegre, ES \\
T. marandobai & $\mathrm{Tma} 1$ & - & - & Montes Claros, MG \\
T. pretiosum & $\mathrm{Tp} 8$ & Helicoverpa zea & Tomate & Afonso Cláudio, ES \\
T. pretiosum & $\mathrm{Tp} 10$ & Trichoplusia ni & Couve & Cristalina, GO \\
T. pretiosum & $\mathrm{Tp} 13$ & - & - & Paraopeba, SP \\
T. pretiosum & $\mathrm{Tp} 15$ & - & - & Fazenda São José, MT \\
T. pretiosum & Tspd & - & - & Piracicaba, SP \\
T. pretiosum & Trecife & Anagasta kuehniella & Repolho & Chã-Grande, PE \\
\hline
\end{tabular}


Gerais, Paraná e São Paulo, verificaram percentagens de parasitismo em ovos de Tuta absoluta que variaram de 43,5 a $69,5 \%$

Não houve diferença significativa na emergência entre as espécies e linhagens de Trichogramma estudadas (Tabela 2), com percentagem média que variou entre $85,9 \mathrm{e} 100 \%$.Aviabilidade está diretamente relacionada às características do hospedeiro como quantidade e qualidade nutricional dos ovos; assim, verificou-se que os ovos de Trichoplusia ni são adequados para o desenvolvimento de todas as espécies estudas, pois são grandes e com valor nutricional adequado ao desenvolvimento dos parasitoides. As viabilidades médias observadas estão de acordo com os resultados de Pratissoli \& Oliveira (1999) e Gonçalves et al. (2003) que avaliaram a viabilidade de Trichogramma pretiosum em ovos de Helicoverpa zea (Boddie) (Lepidoptera: Noctuidae) e Sitotroga cerealella (Oliver) (Lepidoptera: Gelechiidae) e observaram valores de viabilidade superiores a 91,6 e $89 \%$, respectivamente. Entretanto, tais resultados das observações de Pratissoli et al. (2008), que ao avaliar o potencial de T. atopovirilia e T. acacioi para o controle de Plutella xylostella (L.) (Lepidoptera: Plutellidae), obtiveram viabilidades médias de 41,3 e $1,67 \%$, respectivamente.

Houve diferença significativa entre as médias de razão sexual das espécies e linhagens de Trichogramma estudadas (Tabela 2). As espécies com a maior razão sexual foram: T. pretiosum (Tp10, Tp13, Tp15 e Trecife), T. exiguum (Te3), T. acacioi (Tac2) e T. marandobai (Tma1), T. demoraesi (Td1), que apresentaram razão sexual igual a 1, produzindo somente descendentes fêmeas. Entretanto, T. pretiosum (Tp8), T. exiguum (Te1), T. atopovirilia (Tat1) e T. pretiosum (Tspd) obtiveram as menores médias de razão sexual, diferindo das espécies acima citadas. Verifica-se que todas as linhagens e espécies atingiram o índice exigido no controle de qualidade de Trichogramma spp., que é de razão sexual igual ou superior a 0,5 (Amaya Navarro, 1998). No presente trabalho, observou-se que a razão sexual, apesar de diferir entre as espécies e linhagens, foi elevada e na maioria igual a 1. Este atributo pode estar correlacionado ao tamanho do hospedeiro, pois várias pesquisas demonstram que em hospedeiros maiores, a razão sexual originada é bem elevada (Suzuki, et al., 1984; Waage, 1986). Os resultados obtidos no presente trabalho foram superiores aos encontrados por Pratissoli \& Parra (2001), que estudaram linhagens de $T$. pretiosum, criadas em ovos de $T$. absoluta, e obtiveram valores entre 0,90 e 0,60 .

Houve diferença significativa entre as médias do número de indivíduos por ovo, das espécies de Trichogramma estudadas (Tabela 2). T. pretiosum (Tp10, Tp15 e Trecife), T. exiguum (Te3) e T. marandobai (Tma1) não diferiram entre si em relação ao número de indivíduos por ovo, que variou entre 2,24 e 2,41, mas diferiram das demais espécies e linhagens. T. exiguum (Te1) apresentou o menor número de indivíduos por ovo $(1,34)$ e as demais espécies e linhagens tiveram taxas semelhantes (entre 1,57 e 1,92). Todas as espécies e linhagens estudadas obtiveram taxa acima de um indivíduo por ovo do

Tabela 2. Parâmetros biológicos de Trichogramma spp. criados em ovos de Trichoplusia $n i^{(1)}$.

\begin{tabular}{|c|c|c|c|c|}
\hline Espécie (linhagem) & Parasitismo (\%) & Emergência (\%) & Razão sexual & № de indivíduos por ovo \\
\hline T. pretiosum (Tspd) & $53,0 \pm 5,2 \mathrm{a}$ & $93,6 \pm 6,9 \mathrm{a}$ & $0,7 \pm 0,08 b$ & $1,6 \pm 0,1 \mathrm{~b}$ \\
\hline T. pretiosum (Tp8) & $26,6 \pm 8,7 \mathrm{~b}$ & $91,2 \pm 9,0 \mathrm{a}$ & $0,9 \pm 0,09 b$ & $1,5 \pm 0,3 b$ \\
\hline T. pretiosum (Tp10) & $19,3 \pm 6,7 \mathrm{c}$ & $89,0 \pm 13,8 \mathrm{a}$ & $1,0 \pm 0,00 \mathrm{a}$ & $2,3 \pm 0,3 \mathrm{a}$ \\
\hline T. pretiosum (Tp13) & $13,0 \pm 9,4 d$ & $89,3 \pm 14,0 \mathrm{a}$ & $1,0 \pm 0,00 \mathrm{a}$ & $1,8 \pm 0,6 b$ \\
\hline T. pretiosum (Tp15) & $16,0 \pm 6,6 \mathrm{c}$ & $89,8 \pm 13,4 \mathrm{a}$ & $1,0 \pm 0,00 \mathrm{a}$ & $2,2 \pm 0,3 \mathrm{a}$ \\
\hline T. pretiosum (Trecife) & $7,6 \pm 5,3 \mathrm{~d}$ & $92,7 \pm 12,9 \mathrm{a}$ & $1,0 \pm 0,00 \mathrm{a}$ & $2,4 \pm 0,5 \mathrm{a}$ \\
\hline T. acacioi $(\mathrm{Tac} 2)$ & $36,0 \pm 5,7 b$ & $88,8 \pm 10,8 \mathrm{a}$ & $1,0 \pm 0,00 \mathrm{a}$ & $1,5 \pm 0,2 b$ \\
\hline T. atopovirilia (Tat1) & $31,0 \pm 7,8 \mathrm{~b}$ & $93,3 \pm 8,8 \mathrm{a}$ & $0,8 \pm 0,07 b$ & $1,7 \pm 0,2 b$ \\
\hline T. exiguum $(\mathrm{Te} 1)$ & $9,6 \pm 6,9 \mathrm{~d}$ & $100,0 \pm 0,0 \mathrm{a}$ & $0,8 \pm 0,06 \mathrm{~b}$ & $1,3 \pm 0,3 \mathrm{c}$ \\
\hline T. exiguum $(\mathrm{Te} 3)$ & $11,3 \pm 5,5 d$ & $96,0 \pm 8,4 \mathrm{a}$ & $1,0 \pm 0,00 \mathrm{a}$ & $2,2 \pm 0,3 \mathrm{a}$ \\
\hline T. marandobai (Tma1) & $9,6 \pm 6,4 d$ & $91,9 \pm 12,6 a$ & $1,0 \pm 0,00 \mathrm{a}$ & $2,3 \pm 0,5 \mathrm{a}$ \\
\hline T. demoraesi $(\mathrm{Td} 1)$ & $20,6 \pm 6,5 c$ & $85,9 \pm 13,5 \mathrm{a}$ & $1,0 \pm 0,00 \mathrm{a}$ & $1,9 \pm 0,1 \mathrm{~b}$ \\
\hline
\end{tabular}

${ }^{(1)}$ Médias \pm erro-padrão seguidas de letras iguais, na coluna, não diferem entre si pelo teste de $\mathrm{Scott}-\mathrm{Knott}$, a $5 \%$ de probabilidade. Temperatura: $25 \pm 1^{\circ} \mathrm{C}$; umidade relativa: $70 \pm 10 \%$ e fotófase: 12 horas. 
hospedeiro, o que pode resultar em indivíduos de menor tamanho e deformados, em consequência da competição intraespecífica (Suzuki et al., 1984). No entanto, não foram observados indivíduos com tais características, o que evidencia que em ovos de $T$. $n i$ há quantidade suficiente de nutrientes para suportar o desenvolvimento de vários parasitoides. Entretanto, em programas de controle biológico, é desejável a emergência de menor número de parasitoides por ovo, pois maior quantidade de nutrientes estará disponível para gerar indivíduos mais fortes e competitivos. O aumento no número de adultos por ovo poderá reduzir a eficiência de controle, com menor quantidade de ovos parasitados (Beserra et al., 2003). Nava et al. (2007) testaram linhagens de Trichogramma bruni Nagaraja (Hymenoptera: Trichogrammatidae), T. apotovirilia e T. pretiosum, em ovos de Stenoma catenifer Walsingham (Lepidoptera: Elachistidae) e obtiveram taxas variáveis de 1,03 e 1,22, valores inferiores ao observado para $T$. pretiosum por Sá \& Parra (1994), em ovos de Heliothis zea, a $25^{\circ} \mathrm{C}$, que foi de 2,2 parasitoides por ovo do hospedeiro.

Os ovos de Trichoplusia ni são adequados para o desenvolvimento das espécies e linhagens estudadas. A linhagem Tspd de Trichogramma pretiosum apresenta potencial para o controle biológico de Trichoplusia ni, por apresentar o melhor desempenho biológico e ser bem adaptada ao hospedeiro. Entretanto, para a continuidade da seleção de espécies e linhagens de Trichogramma, para o manejo fitossanitário de $T$. $n i$, as etapas de semi-campo e campo devem ser realizadas.

\section{Conclusões}

1. Os ovos de Trichoplusia ni são adequados para o desenvolvimento de espécies e linhagens de Trichogramma.

2. A linhagem Tspd de $T$. pretiosum apresenta o melhor potencial para o manejo fitossanitário de Trichoplusia $n i$ entre as espécies e linhagens estudadas.

\section{Agradecimentos}

À Coordenação de Aperfeiçoamento de Pessoal de Nível Superior, por concessão de bolsa; ao Conselho Nacional de Desenvolvimento Científico e Tecnológico, pelo suporte financeiro.

\section{Referências}

AMAYA NAVARRO, M. Trichogramma spp.: producción, uso y manejo en Colombia. Guadalajara de Buga: Impretec, 1998. 176p.

BESERRA, E.B.; DIAS, C.T. dos S.; PARRA, J.R.P. Características biológicas de linhagens de Trichogramma pretiosum desenvolvidas em ovos de Spodoptera frugiperda. Acta Scientiarum, v.25, p.479-483, 2003.

CASTELLS, E.; BERENBAUM, M.R. Resistance of the generalist moth Trichoplusia ni (Noctuidae) to a novel chemical defense in the invasive plant Conium maculatum. Chemoecology, v.18, p.11-18, 2008.

CÔNSOLI, F.L.; PARRA, J.R.P. Egg laying and development of different strains of Trichogramma pretiosum Riley (Hymenoptera: Trichogrammatidae) in artificial eggs. Anais da Sociedade Entomológica do Brasil, v.28, p.173-177, 1999.

GODIN, C.; BOIVIN, G. Lepidopterous pests of Brassica crops and their parasitoids in southwestern Quebec. Environmental Entomology, v.27, p.1157-1165, 1998.

GONÇALVES, J.R.; HOLTZ, A.M.; PRATISSOLI, D.; GUEDES, R.N.C. Avaliação da qualidade de Trichogramma pretiosum (Hymenoptera: Trichogrammatidae) em ovos de Sitotroga cerealella (Lepidoptera: Gelechiidae). Acta Scientiarum. Agronomy, v.25, p.485-489, 2003.

GREENE, G.L.; LEPPLA, N.C.; DICKERSON, W.A. Velvetbean caterpillar: rearing procedure and artificial medium. Journal of Economic Entomology, v.69, p.487-488, 1976.

HART, K.A.; PIMENTEL, D. Environmental and economic costs of pesticide use. In: PIMENTEL, D. (Ed.). Encyclopaedia of pest management. New York: Marcel Dekker, 2002. p.237-239.

JANMAAT, A.F.; MYERS, J. Rapid evolution and the cost of resistance to Bacillus thuringiensis in greenhouse populations of cabbage loopers, Trichoplusia ni. Proceedings of the Royal Society of London, v.270, p.2263-2270, 2003.

JOST, D.J.; PITRE, H.N. Soybean looper and cabbage looper (Lepidoptera: Noctuidae) populations in cotton and soybean cropping systems in Mississippi. Journal of Entomological Science, v.37, p.227-235, 2002.

LIU, T.X.; SPARKS JUNIO, A.N.; CHEN, W.; LIANG, G.M.; BRISTER, C. Toxicity, persistence, and efficacy of indoxacarb on cabbage looper (Lepidoptera: Noctuidae) on cabbage. Journal of Economic Entomology, v.95, p.360-367, 2002.

MANSFIELD, S.; MILLS, N.J. A comparison of methodologies for the assessment of host preferences of the gregarious egg parasitoid Trichogramma platneri. Biological Control, v.29, p.332-340, 2004.

NAVA, D.E.; TAKAHASHI, K.M.; PARRA, J.R.P. Linhagens de Trichogramma e Trichogrammatoidea para controle de Stenoma catenifer. Pesquisa Agropecuária Brasileira, v.42, p.9-16, 2007.

PARRA, J.R.P. Criação massal de inimigos naturais. In: PARRA, J.R.P.; BOTELHO, P.S.M.; CORRÊA-FERREIRA, B.S.; BENTO, J.M.S. (Ed.). Controle biológico no Brasil: parasitóides e predadores. São Paulo: Manole, 2002. p.143-161. 
PRATISSOLI, D.; OLIVEIRA, H.N. de. Influência da idade dos ovos de Helicoverpa zea (Boddie) no parasitismo de Trichogramma pretiosum Riley. Pesquisa Agropecuária Brasileira, v.34, p.891-896, 1999.

PRATISSOLI, D.; PARRA, J.R.P. Seleção de linhagens de Trichogrammapretiosum Riley(Hymenoptera: Trichogrammatidae) para o controle das traças Tuta absoluta (Meyrick) e Phthorimaea operculella (Zeller) (Lepidoptera: Gelechiidae). Neotropical Entomology, v.30, p. 277-282, 2001.

PRATISSOLI, D.; POLANCZYK, R.A.; HOLTZ, A.M.; DALVI, L.P.; SILVA, A.F.; SILVA, L.N. Selection of Trichogramma species for controlling the Diamondback moth. Horticultura Brasileira, v.26, p.259-261, 2008.

PRATISSOLI, D.; POLANCZYK, R.A.; PEREIRA, C.L.T.; FURTADO, I.S. de A.; COCHETO, J.G. Influência da fase embrionária dos ovos da traça-das-crucíferas sobre fêmeas de Trichogramma pretiosum com diferentes idades. Horticultura Brasileira, v.25, p.286-290, 2007.

RORIZ, V.; OLIVEIRA, L.; GARCIA, P. Host suitability and preference studies of Trichogramma cordubensis (Hymenoptera: Trichogrammatidae). Biological Control, v.36, p.331-336, 2006.
SÁ, L.A.N. de; PARRA, J.R.P. Biology and parasitism of Trichogramma pretiosum Riley (Hym., Trichogrammatidae) on Ephestia kuehniella (Zeller) (Lep., Pyralidae) and Heliothis zea (Boddie) (Lep., Noctuidae) eggs. Journal of Applied Entomology, v.118, p.38-43, 1994.

STEIN, C.P.; PARRA, J.R.P. Aspectos biológicos de Trichogramma spp. em diferentes hospedeiros. Anais da Sociedade Entomológica do Brasil, v.16, p.163-171, 1987.

SUZUKI, Y.; TSUJI, H.; SASAKAWA, M. Sex allocation and effects of superparasitism on secondary sex ratios in the gregarious parasitoid, Trichogramma chilonis (Hymenoptera: Trichogrammatidae). Animal Behavior, v.32, p.478-484, 1984.

VINSON, S.B. Comportamento de seleção hospedeira de parasitóides de ovos, com ênfase na família Trichogrammatidae. In: PARRA, J.R.P.; ZUCCHI, R.A. (Ed.). Trichogramma e o controle biológico aplicado. Piracicaba: Fealq, 1997. p.67-120.

WAAGE, J.K. Family planning in parasitoids: adaptive patterns of progeny and sex allocation. In: WAAGE, J.K.; GREATHEAD, D. (Ed). Insect parasitoids. London: Academic, 1986. p.63-95.

Recebido em 6 de abril de 2009 e aprovado em 22 de setembro de 2009 